EPJ Web of Conferences 41, 05015 (2013)

DOI: $10.1051 /$ epjconf/20134105015

(C) Owned by the authors, published by EDP Sciences, 2013

\title{
New perspectives on ultrafast Förster Resonant Energy Transfer
}

\author{
I. Pugliesi ${ }^{1}$, H. Langhals ${ }^{2}$, H. Kauffmann ${ }^{3}$ and E. Riedle ${ }^{1}$ \\ ${ }^{1}$ LS für BioMolekulare Optik, LMU München, 80538 München, Germany \\ ${ }^{2}$ Department für Chemie, LMU München, 81377 München, Germany \\ ${ }^{3}$ Faculty of Physics, University of Vienna, 1090 Vienna, Austria
}

\begin{abstract}
We show that perylene diimide dyads based on a donor-spacer-acceptor motif violate Förster's dipole-dipole interaction picture for energy transfer in the low picosecond to sub-100 femtosecond regime. First theoretical explanations are presented.
\end{abstract}

\section{Perylene bisimide dyads reveal new energy transfer mechanisms}

We present an in-depth investigation of the very principles of Förster Resonant Energy Transfer (FRET) by pump-probe and 2D electronic spectroscopy, chemical variation and ab initio calculations on an extended set of perylene diimide dyads based on a donor-spacer-acceptor motif (as shown in figure 1 and 2). These dyads have revealed many unexpected deviations from the behaviour predicted by the simple dipole-dipole interaction picture used to describe FRET. These surprising results range from unexpectedly fast FRET rates going far below 400 fs to picosecond energy transfer in dyads with orthogonal transition dipoles and deviations from the $1 / \mathrm{R}^{6}$ donor-acceptor distance dependence. For dyads with electron donating spacers that quench the fluorescence of the energy donor, the electron transfer transition itself mediates FRET.

FRET has become a process of ubiquitous importance in chemistry and biochemistry. While proximity measurements of light absorbing and fluorescent structures still rely on the basic theory of FRET, our results show that a more refined model is required for an accurate description of this photophysical process.

\section{Ultrafast FRET in dyads with parallel transition moments: strong electronic and vibrational mixing}

Transient absorption measurements of the perylene-terylene diimide dyad with parallel transition dipole moments shown in figure 1 (middle) have been carried out with the pump-probe setup described in ref [1]. The spectral changes in the NIR, where donor and acceptor have distinct absorption bands at 820 and $880 \mathrm{~nm}$ (see figure 1b), reveal an ultrafast energy transfer below $400 \mathrm{fs}$. To further elucidate the mechanism behind this ultrafast FRET, 2D electronic spectra were recorded with the setup described in ref [2]. The 2D spectrum in figure 1c reveals that the onset of the stimulated emission occurs for as early a waiting time as $30 \mathrm{fs}$ (see figure 1c, white arrow) and 2D spectra recorded at longer waiting times indicate that the FRET is as fast as $50 \mathrm{fs}$. While in the pump-probe experiments the NOPA pulse used for excitation only covers the highest vibronic band

This is an Open Access article distributed under the terms of the Creative Commons Attribution License 2.0, which permits unrestricted use, distribution, and reproduction in any medium, provided the original work is properly cited. 
of the donor absorption, in the 2D measurements the ultrabroad 9 fs VIS NOPA pulses covers all vibronic bands of the donor absorption and parts of the absorption of the acceptor $(530-720 \mathrm{~nm})$. The broader pulse causes a speed up in the energy transfer and indicates that the process is excitation dependent. The strong mixing of the electronic and vibrational degrees of freedom, which has been chemically engineered into this dyad, has to be taken into account in order to rationalise this behaviour.
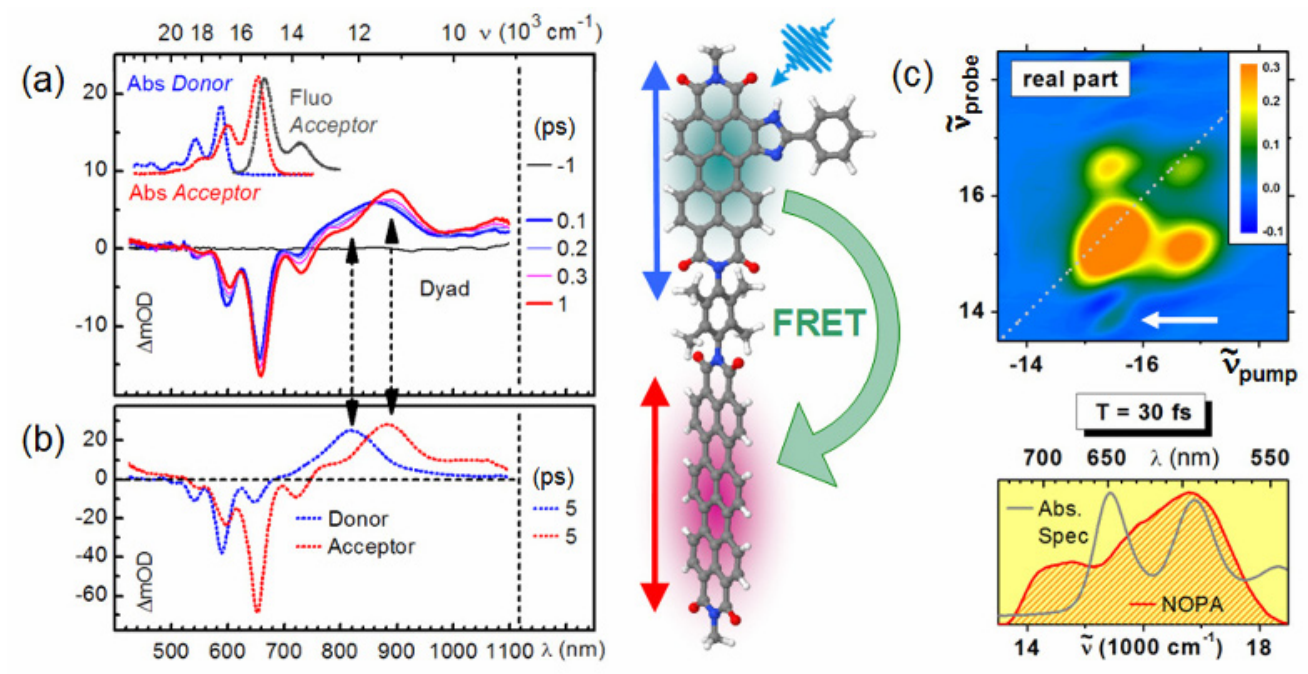

Fig. 1. (a) Transient absorption spectra of the perylene-terylene diimide dyad shown in the centre together with (b) the transient absorption spectra of the pure donor and acceptor. (c) 2D spectrum of the dyad for a waiting time $\mathrm{T}$ of $30 \mathrm{fs}$. The onset of the stimulated emission of the energy acceptor is indicated with a white arrow.

\section{FRET in dyads with orthogonal transition moments: violation of the $\mathbf{R}^{6}$ distance dependence}

Transient absorption measurements in chloroform of the dyads shown in figure 2 reveal that rapid FRET from the benzoperylene (donor) to the perylene (acceptor) moiety is occurring although the transition dipole moments are orthogonal to each other. According to the point dipole approximation used in Förster theory no energy transfer should occur in an orthogonal arrangement. Experimentally we do, however, observe ultrafast transfer times ranging from 9 ps up to 54 ps depending on the spacer length (see Figure 2). The determination of the Coulombic coupling via the transition density cube approach furthermore reveals that in the orthogonal arrangement the higher multipole moments are not responsible for the energy transfer.

In further pump-probe experiments we observe that the energy transfer rate increases upon increase of the sample temperature [3]. This temperature dependence can only be simulated with an extended Förster model [4] that includes low wavenumber ground state vibrations and more importantly structural and electronic deformations induced by solvent fluctuations. These deformations break the orthogonal arrangement of the dipoles and enable the ultrafast and near unity efficiency energy transfer.

Fermi's Golden Rule rate for this noise-induced FRET mechanism predicts a donor-acceptor distance dependence on the energy transfer rate that scales with $\left(1 / \mathrm{R}^{3}\right)$. This is in contrast to the standard FRET $\left(1 / \mathrm{R}^{6}\right)$ dependence. The time-resolved measurements in figure 2 indeed deviate from the $\left(1 / R^{6}\right)$ behaviour and point towards a much weaker distance dependence that is better described by $\left(1 / \mathrm{R}^{3}\right)$. 

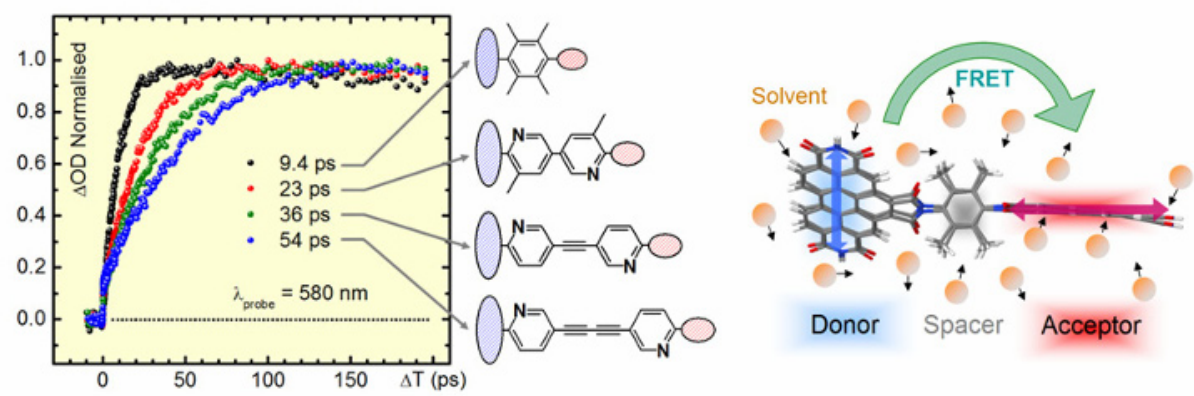

Fig. 2. Distance dependence of the FRET times for perylene diimide dyads with orthogonal transition dipoles and varying spacer length. The cartoon on the right hand side depicts the mechanism mediating the energy transfer.

\section{Electron transfer induces FRET}
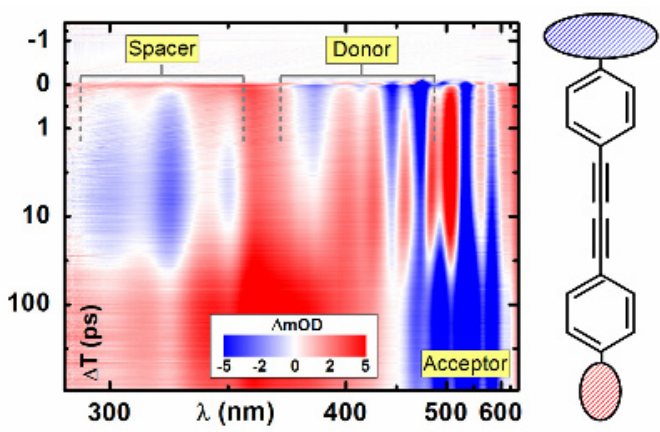

Fig. 3. Transient absorption spectrum of a perylene diimide dyad with a diphenylbutadiyne spacer.

Figure 3 shows the transient absorption spectrum of a perylene diimide dyad with a diphenylbutadiyne spacer. Unlike the dyads discussed in sections 2 and 3 , here the spacer participates in the energy transfer dynamics. The ground state bleach features of the spacer located between 300 and $350 \mathrm{~nm}$ appear with a time constant of 4 ps and decay with $35 \mathrm{ps}$, the same time constant present in the rise of the ground state bleach and stimulated emission of the perylene energy acceptor. The appearance of the spacer bleach signal is due to electron transfer from the spacer to the energy donor. Although this process quenches the fluorescence of the donor it does not inhibit energy transfer to the perylene energy acceptor, as can be seen in the transient measurement in figure 3. The transient signatures reveal that electron and energy transfer are sequential and indicate that the donor-spacer electron transfer state itself is responsible for the energy transfer, probably via multipole-multipole interactions with the locally excited state of the acceptor. Thus this dyad represents a first example that shows how electron transfer can be connected to energy transfer for the use in novel photovoltaic and optoelectronic devices.

\section{References}

1. U. Megerle, I. Pugliesi, C. Schriever, C. Sailer, E. Riedle, Appl. Phys. B, 96, 215 (2009)

2. F. Milota, J. Sperling, A. Nemeth, H. F. Kauffmann, Chem. Phys. 357, 45 (2009)

3. H. Langhals, A. J. Esterbauer, A. Walter, E. Riedle, and I. Pugliesi, J. Am. Chem. Soc. 132, 16777, (2010)

4. P. Nalbach, I. Pugliesi, H. Langhals and M. Thorwart, Phys. Rev. Lett. 108, 218302 (2012) 